\title{
Implementation of Augmented reality in CAD design
}

\author{
Andrijana Bocevska \\ Faculty of Information and Communication Technologies, Partizanska bb, \\ 7000 Bitola, Macedonia \\ andrijana.bocevska@uklo.edu.mk
}

\begin{abstract}
The visual representation of a product and the role of visualization have recently become a central issue in design research. By enhancing a real scene with computer generated objects, Augmented Reality (AR), has proven itself as a valuable Human-Computer Interface (HCI) in numerous application areas such as medical, military, entertainment and manufacturing. Also AR has potentials in design because the current interface provided by Computer-Aided Design (CAD) packages is less intuitive and reports show that the presence of physical objects help design. In this paper we proposed approach that user can create own 3D augmented reality scenes which enables interaction between real world and virtual object at the same time. For this purpose BuildAR software is applied using marker-based camera tracking and $3 \mathrm{D}$ object is obtained with standard CAD system SolidWorks.
\end{abstract}

Keywords: augmented reality, 3D CAD, marker.

\section{Introduction}

Current Computer Aided Design (CAD) systems offer extremely rich modeling features and functions which increase the productivity of new products design. Although the geometrical database has been 3D for a long time, the user interaction made through keyboard, mouse and CRT/LCD display which are only 2D devices with CAD systems has not been significantly changed.

Augmented Reality $(A R)$ is a field of computer research which deals with the combination of real world and computer generated data. Unlike Virtual Reality (VR) systems, in which users are completely immersed in the virtual environment, AR users see the virtual objects and the real world co-existing in the same space. It is the goal of AR to supplement reality rather than completely replace it as in conventional VR applications. The AR technology provides useful information about the environment enhancing perception and interaction with the real world. The user is able to manipulate both real objects, and the virtual ones.

Augmented Reality provides new perspectives for user interaction with Computer Aided Design (CAD) tools. AR enhances the perception of 3D objects by co-location, leading to information with less perceptive ambiguities. The users have the possibility to translate, rotate and scale the overlaid 3D CAD model by using a tangible user interface composed from indicative markers. 
Augmented Reality also is known as Mixed Reality (MR), according to [1], Fig.1. Being characteristic of integration of virtual and real scene many application of Augmented Reality are emerging in the fields of education, medical treatment, entertainment, design, etc.

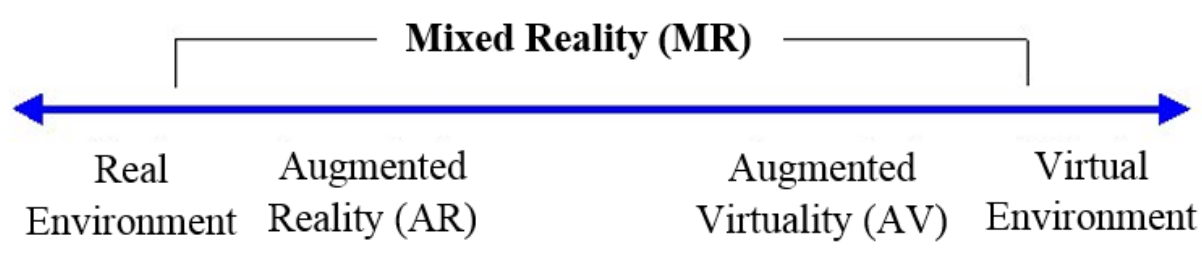

Fig. 1. Simplified representation of a reality-virtuality (RV) continuum.

\subsection{Types of augmented reality}

The characteristics of AR systems can be further understood from three classical and widely used criteria [2]:

- Combines virtual and real - AR requires display technology that allows the user to simultaneously see virtual and real information in a combined view. Traditional displays can show only computer-generated images and are thus insufficient for AR.

- Registered in 3-D - AR relies on an intimate coupling between the virtual and the real that is based on their geometrical relationship. This makes it possible to render the virtual content with the right placement and 3D perspective with respect to the real.

- Interactive in real time - the AR system must run at interactive frame rates, such that it can superimpose information in real-time and allow user interaction.

The basic components in AR applications are a display, a camera and a computer with application software [3]. Different kinds of hardware can be used to implement this, e.g. camera phones, handheld computers, laptops, headmounted displays (HMD) etc. There are two types of augmented reality [4]:

- Marker based - different type of augmented reality markers are images that can be detected by a camera and used with software as the location for virtual assets placed in a scene. A camera is used with AR software to detect augmented reality markers as the location for virtual objects. The result is that an image can be viewed, on a scene and digital assets are placed into the scene at the location of the markers.

- Marker less: In marker less AR the image is gathered through internet and displayed on any specific location i.e. by using GPS. This application does not require marker to display the content. 


\section{Markers}

Markers are square patterns you print out and display to the camera. The AR software uses computer vision software to recognize the patterns and calculate the position of the real camera relative to the printed pattern. Once the position of the real camera is known, then a 3D model can be drawn relative to the real marker. 3D models are virtual objects that will appear to be attached to the markers, creating augmented reality experience.

Printed markers usually are black and white squares with a thick border, and a unique pattern in the center. Different markers have different patterns inside. There should be enough white space around the outside of the black square so that the marker can be easily found by the computer vision software. The pattern in the middle of the square should be twice the width of the boarder of the square. The BuildAR software has two markers shown on Fig. 2, but you can also create your own marker.
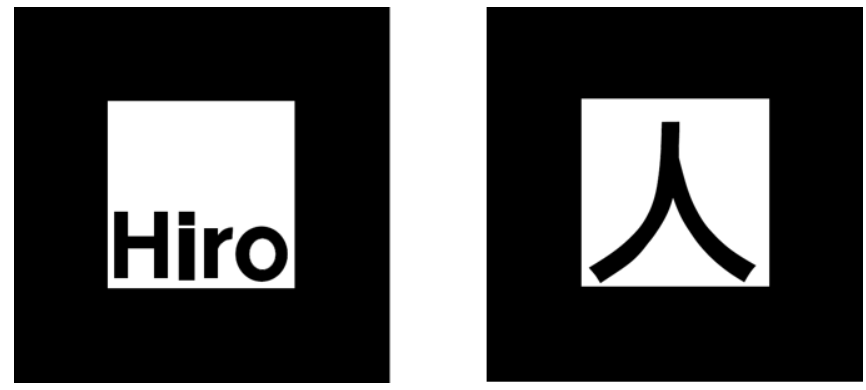

Fig. 2. Examples of AR markers.

\section{CAD system SolidWorks to AR methodology}

For integrating the CAD system SolidWorks and augmented reality, BuildAR software is applied. BuildAR uses marker-based camera tracking, which means that the $3 \mathrm{D}$ models appear attached to physical printed marker. Augmented reality scene can be build by creating a set of markers and a set of 3D models. 3D models can be created in almost any modelling program or download them from the internet. Also BuildAR provides an easy to use graphical user interface that simplifies the process of creating AR scenes, meaning that you do not need to be a programmer or computer expert to create AR scenes. BuildAR is a product of the Human Interface Technology Lab NZ, based at the University of Canterbury, in Christchurch, New Zealand.

The CAD to AR methodology involves the next steps:

- Generate pattern in BuildAR. Before tracking patterns can be produced the images must previously have been made in an image editing tool and save it as a 16 colour bitmap file for use. The created marker for this methodology use text as an image. 
- Load model onto marker. 3D model has been developed with CAD system SolidWorks. In order to use in BuildAR it must be translated in one of the support following file formats: 3DS, LWO, OBJ, STL, FLT. The recommended file format is the OpenSceneGraph .ive file format. Other file formats can be used, but the quality of the importers for these other formats varies, sometimes they can produce strange normals, or missing textures/animations. For the CAD model data translation STL format is applied.

- Move, rotate and scale the model. If we want to adjust the way the 3D model appears on the marker we use the manipulation control panel and type in new positions, rotations and scales manually or simply click on the 3D object in the live view and use the 3D widget buttons on the toolbar. At the same time the scene tree shows which markers and 3D objects currently make up the scene. The tree is a list of markers, each with an object underneath them. Markers and object can be added or deleted from the Scene Tree. If we want to add more markers and 3D objects to augmented reality scene these steps must be repeated.

- Save and load the augmented reality scene. After editing the scene it can be saved to an XML file.

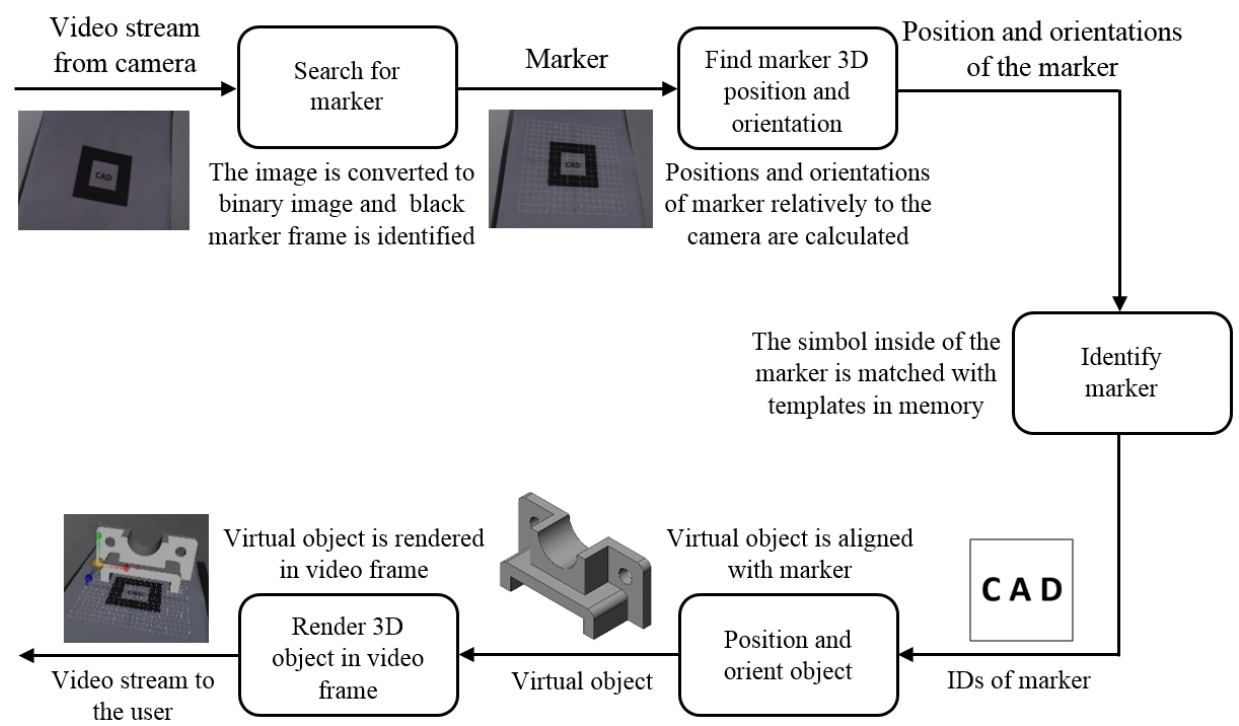

Fig. 3. The process of video-based marker detection and overlay of virtual object.

The augmentation process is as follows:

1. The video camera captures video of the real world and sends it to the computer.

2. The live video image is turned into a binary (black or white) image based on a lighting threshold value.

3. This image is then searched for square regions. BuildAR software finds all the squares in the binary image. 
4. For each square, the pattern inside the square is captured and matched against some pretrained pattern templates.

5. BuildAR software then uses the known square size and pattern orientation to calculate the position of the real video camera relative to the physical marker. BuildAR software computes the transformation matrix that describes the position, rotation, and size of an object.

6. The virtual object is thereafter located in relation to that marker. Thus the user experiences augmented reality, seeing the real world through the real time video with virtual models.

The diagram in Figure 3 shows the image processing, and in Figure 4 shows an sample marker in frame without virtual objects and sample marker in frame with virtual object.
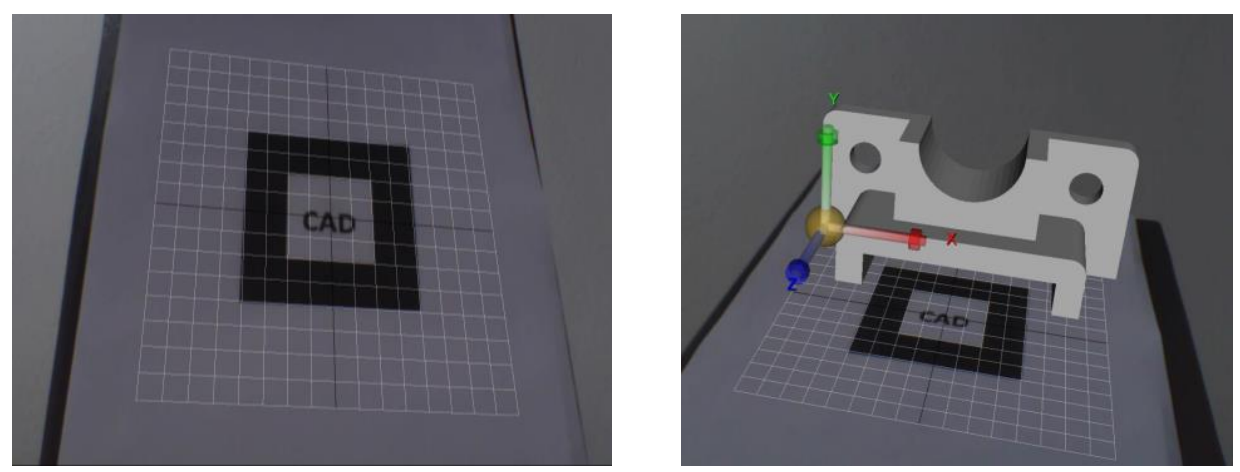

Fig. 4. Sample marker in frame without and with virtual object.

\section{Conclusion}

Marker based augmented reality technologies represent useful tools to visualize and interact with 3D CAD models. The advantages of this application are that it would display object in 3D and enable the user to translate, rotate and scale the overlaid 3D CAD model by using a printable marker. We see presence of AR in many aspects of everyday living. This paper presented how it is possible to use augmented reality technologies in CAD design i.e. the possibility user to view the virtual object in the real world using a marker based AR system. For the CAD model data translation to the AR software STL format was applied. Our opinion is that this application play an important role in design visualization and can be used in achiving proper understanding of the design. 


\section{References}

1. Milgram, P., Takemura, H., Utsumi, A., Kishino, F., Augmented reality: a class of displays on the reality-virtuality continuum, SPIE, Telemanipulator and Telepresence Technologies, 2351. (1994)

2. Azuma, R. T. A survey of augmented reality. Presence: Teleoperators and Virtual Environments, 6(4):355-385. (1997)

3. Azuma, R., Baillot, Y., Behringer, R., Feiner, S., Julier, S., MacIntyre, B.: "Recent advances in augmented reality", IEEE Computer Graphics and Applications, 21 (2001), no. 6, pp. 34- 47. (2001)

4. Raviraj, S. P., Marker Based Augmented Reality Using Android OS, Pune University -ISSN2277128X, Vol. 3, No. 5. (2013)

5. Siltanen, S.: Theory and applications of marker-based augmented reality. ISBN 978-951- 387450-6 (URL: http://www.vtt.fi/publications/index.jsp) ISSN 2242-1203 (URL: http://www.vtt.fi/publications/index.jsp) Copyright@ VTT (2012)

6. Pasman, W., Woodward, C.: Implementation of an augmented reality system on a PDA. Proceeding of the Second IEEE and ACM International Symposium on Mixed and Augmented Reality (ISMAR 2003), Tokyo, Japan, 276-277. (2003) 